\title{
Thermal characterisation of bio-based building materials
}

\author{
S. Costanzo ${ }^{1}$, A. Cusumano ${ }^{1}$, C. Giaconia ${ }^{1} \&$ G. Giaconia ${ }^{2}$ \\ ${ }^{1}$ Department of Energetics and Environmental Researches, \\ University of Palermo, Italy \\ ${ }^{2}$ Department of Civil Engineering, University of Messina, Italy
}

\begin{abstract}
The characterisation of indigenous renewable biomass sources applicable for making bio-based building (BBB) materials is crucial as it can significantly affect the cost of production and the quality of material. Nowadays in any specific area, wide ranges of biomass sources are usually available but only a few sources may be suitable for making BBB material. Therefore the suited sources should be determined. In this paper, the use of BBB materials is focused on Insulation Materials which are widely used in the buildings. Compared with currently used materials, these materials are bio-degradable. Insulation material with a good energy-adsorbing property and binder material have been developed as a mixture of renewable biomass and concrete under certain of reaction conditions. In detail, the BBB material can contribute to energy saving in terms of consumption of low energy during its production process, as well as of degradability. The experimental assessment of non steady-state thermal characteristics of a BBB material is presented here. The results will be useful to develop a database of the physical properties available for the building industry. Keywords: bio-based materials, sustainable materials, thermal properties.
\end{abstract}

\section{Introduction}

Studies concerning the revaluation and use of human and natural resources and their contribution to social economy and ecology are of paramount importance in modern urban planning. Bio-based materials are an important natural resource 
for construction purposes and they are more and more taken into account for alternative sustainable components. Studies about sustainable architectural solutions and construction techniques are still in progress at the Department of Energetics and Environmental Researches (DREAM) of the Palermo University, both by using natural raw materials and by finding new derived products and mixed systems (comprising preservation treatments, combinations with different kinds of lightweight concrete, with raw earth and other bio-materials, etc.). Design of building products and components made of bio-based materials produced in humid climate Countries is presently addressed to possible European markets and in Far East market as well. In this context, besides the characterization of building products and components made of bio-based materials, physical analysis and characterisation of thermal performances of the bio-based materials can be carried out using a Test facility available at the DREAM. The Bio Based Building Materials are based on natural resources and are environmental friendly during the manufacturing process as well as in the wasting phase at the end of its life cycle. They are characterized by:

- Low cost of the raw material (waste matters)

- Low production cost (can be manufactured by the end user)

- Low handling cost (can be manufactured on the spot)

- $\quad$ Low dumping cost at the life end (can be recycled)

Nowadays only a small amount of civil infrastructures in developed countries make us of BBBMs because of the high handling and production costs. They, instead, shall be widely used in developing countries where the cost of labour is very low and the production and using points are very close. Research programs upon composite materials made of biological components aimed to the building application are already in progress all over the world [1-3]. An in-depth study of Lamy and Baley [4] deals with the stiffness of epoxy panels filled with flax fibres. A comparative analysis upon the improvement of the mechanical characteristics of polyester resins filled with natural fibres of various kind was carried out by De Albuquerque et al [5]. Studies on polymers strengthened with natural fibres were also carried out by Mohanty et al [6] and by Williams and Wool [7]. More recent papers deal with Bio-Composites sustainability [8-11]. Whereas the mechanical and physical properties of the BBBMs are widely known, we can't say the same for the thermal ones. An in-depth knowledge of the thermal properties of the building materials is indeed crucial for the assessment of its energy balance and thermal performances and the studies ought to be carried out through ever more accurate computer programs for the simulation of non-steady state thermal behaviour of buildings. It is evident that a sophisticated computer program needs as much exact coefficients, but precise values of the thermal capacity as well as of the thermal effusivity and diffusivity are not available for all type of materials. This problem is more evident for materials which are innovative or made by mixing diverse percentages of diverse materials.

In this paper, the experimental assessment of the thermo-physical properties of a particular BBBM, carried out by the authors within the laboratories of the DREAM is presented. This BBB material has been produced by the School of 
Environment Science \& Technology, Tian Jin University, Popular Republic of China, within the International project "Toward a Better Environment: Implementation of Energy-Saving Buildings in China", Asia Pro Eco (ENER-C)".

\section{Measurement methodology}

The BBB materials examined in this paper are made by mixing ordinary concrete with wastes of rice manufacturing which are widely available in China at very low cost. The physical characteristics of the materials used in this study are reported in table 1. For each sample three specimens have been obtained. The physical quantities reported in the tables have to be considered as mean values over three measures.

Table 1: Physical characteristics of the materials.

\begin{tabular}{|c||c|c|c|c|c|}
\hline $\begin{array}{c}\text { Sample } \\
\text { Code }\end{array}$ & $\begin{array}{c}\text { Weight } \\
{[\mathrm{gr}]}\end{array}$ & $\begin{array}{c}\mathrm{X} \\
{[\mathrm{mm}]}\end{array}$ & $\begin{array}{c}\mathrm{Y} \\
{[\mathrm{mm}]}\end{array}$ & $\begin{array}{c}\mathrm{Z} \\
{[\mathrm{mm}]}\end{array}$ & $\begin{array}{c}\text { Density } \\
{\left[\mathrm{kg} / \mathrm{m}^{3}\right]}\end{array}$ \\
\hline \hline $1-3$ & 512,822 & 39,3 & 40,0 & 160,0 & 2038,89 \\
\hline $4-1$ & 243,600 & 39,5 & 40,0 & 160,0 & 963,61 \\
\hline $11-3$ & 227,722 & 39,5 & 40,0 & 159,0 & 906,46 \\
\hline $2-1$ & 206,948 & 39,5 & 39,5 & 160,0 & 828,99 \\
\hline $3-2$ & 176,131 & 39,5 & 40,0 & 160,0 & 696,72 \\
\hline $12-1$ & 170,050 & 39,7 & 39,5 & 160,0 & 677,75 \\
\hline Samples were dried up for two hours in oven and stabilized in dry ambient for two hours \\
\hline
\end{tabular}

The sketch in Figure 1 shows the layout of one half of the test equipment used by the authors for measuring the thermal characteristics of the BBB samples provided by the Tianjin University.

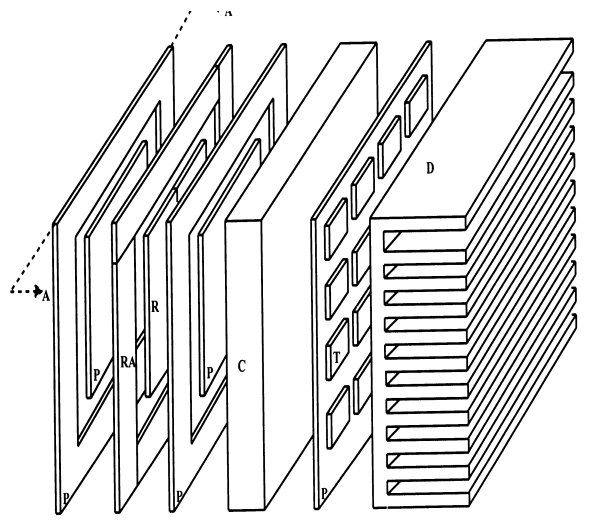

Legend:

$\mathrm{P}=$ Equalizer Plate \& Guard Ring

$\mathrm{R}=$ Main Heater

RA $=$ Heated Guard Ring

$\mathrm{C}=$ Sample

$\mathrm{T}=$ Thermoelectric Heat Pump

$\mathrm{D}=$ Cooler

Figure 1: Layout of the measuring equipment. 
The equivalent circuit of one half of the measuring equipment schematized in fig. 1 is reported in fig. 2 .

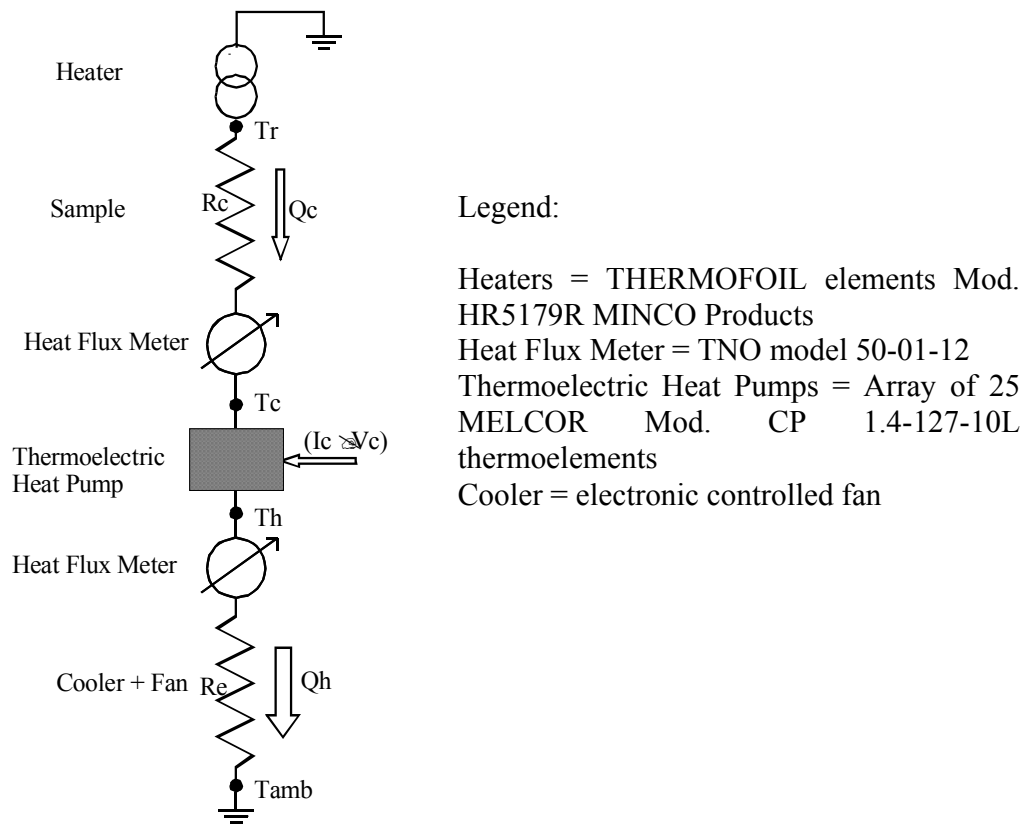

Figure 2: Equivalent circuit of the measuring equipment.

Fig. 3 depicts the overall assembly of the measuring instrumentation. It is possible to single out four power supplies, two of them used for the main heaters and for the guard rings, the others used for feeding a constant current into the thermoelements.

Power supplies are controlled by a Desktop Computer through an IEEE 488 interface and a standard RS232 serial interface. The computer, via a proprietary interface, is capable of acquire all the relevant data (referring to the thermal and electrical quantities) and, using a BASIC program specifically designed and implemented, carries out the control of the thermoelements.

The measures of thermal conductivity were carried out in accordance with the following protocol: the thermoelectric refrigerator was set on and regulated for the minimum possible rate, waiting for the steady state condition; when all the temperatures have been stabilized for a period of almost one hour, the heating plate was energized.

After a transient period a new steady state conditions were reached and maintained for almost half an hour. 


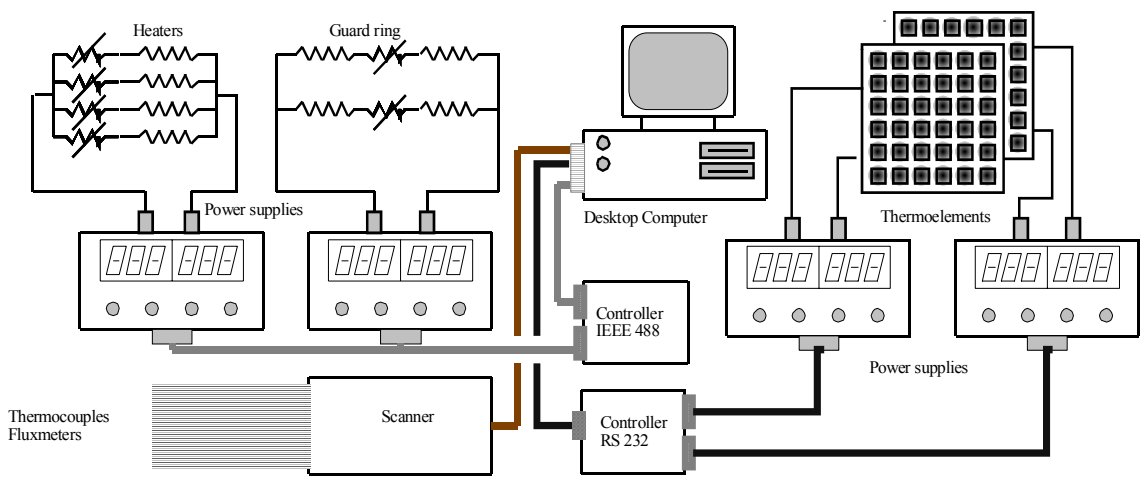

Figure 3: The measuring instrumentation.

From the knowledge of the temperature difference between the two faces and of the thermal flux through the sample, the thermal conductivity of the material was calculated

$$
\frac{\Phi}{S}=\frac{\chi}{S} \Delta T \quad\left[\text { Watt } / m^{2}\right]
$$

Looking at fig. 4 it is possible to note the transferring time delay of the thermal flux between the faces of the sample.

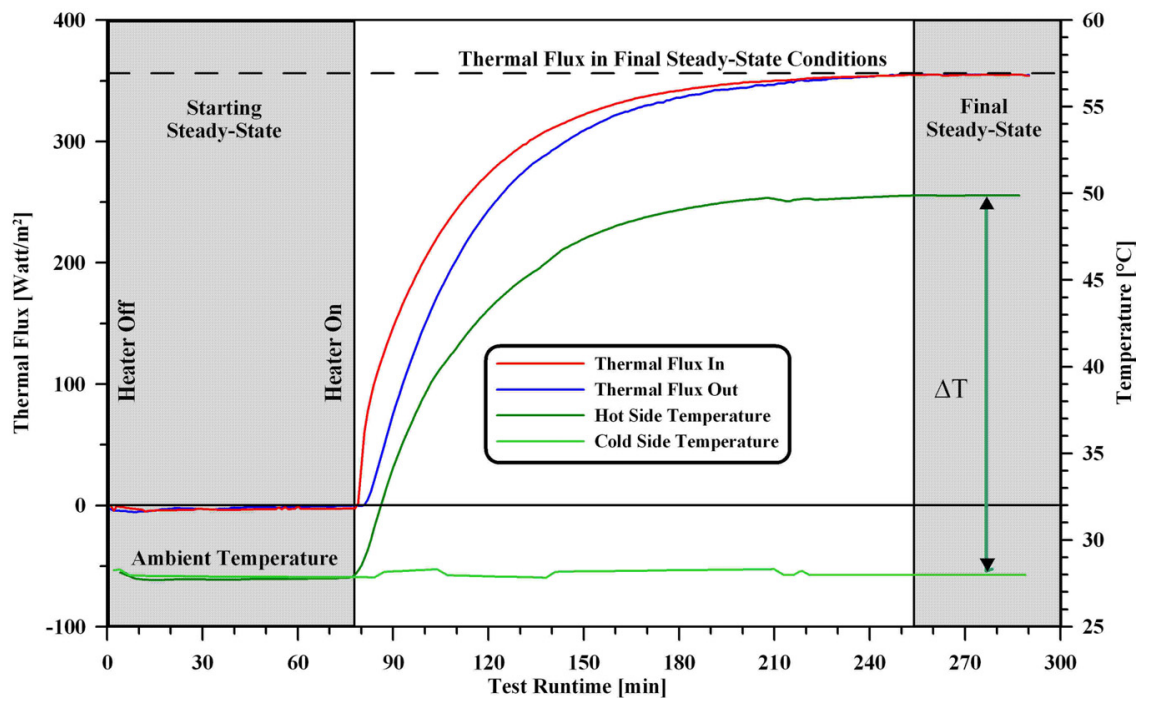

Figure 4: Example of a test run. 
This time lag is tied to the heath transmission velocity through a medium having thermal resistance and thermal capacity, quantities that one can resume in the thermal diffusivity parameter, say $\alpha(2)$ :

$$
\alpha=\chi / \gamma c_{p} \quad\left[m^{2} / \mathrm{sec}\right]
$$

The results of thermal conductivity measurements carried out on the six types of samples are reported in table 2 .

Table 2: Thermo-physical characteristics of the samples in steady state.

\begin{tabular}{|c|c|c|c|c|c|c|}
\hline $\begin{array}{c}\text { Sample } \\
\text { Code }\end{array}$ & $\begin{array}{c}\text { Weight } \\
{[\mathrm{Kg}]}\end{array}$ & $\begin{array}{c}\text { Volume } \\
{\left[\mathrm{m}^{3}\right]}\end{array}$ & $\begin{array}{c}\text { Density } \\
{\left[\mathrm{kg} / \mathrm{m}^{3}\right]}\end{array}$ & $\begin{array}{c}\% \\
\text { BBBM }\end{array}$ & $\begin{array}{c}\chi \\
{[\text { Watt } / \mathrm{mK}]}\end{array}$ & $\begin{array}{c}\text { Standard } \\
\text { Deviation }\end{array}$ \\
\hline $1-3$ & 0.51282 & 0.000252 & 2038.89 & 40 & 0.5384 & 0.02118 \\
\hline $4-1$ & 0.24360 & 0.000253 & 963.61 & 60 & 0.2125 & 0.02310 \\
\hline $11-3$ & 0.22772 & 0.000251 & 906.46 & 65 & 0.2115 & 0.01679 \\
\hline $2-1$ & 0.20695 & 0.000250 & 828.99 & 70 & 0.1810 & 0.01975 \\
\hline $3-2$ & 0.17613 & 0.000253 & 696.72 & 75 & 0.1725 & 0.01879 \\
\hline $12-1$ & 0.17005 & 0.000251 & 677.75 & 80 & 0.1390 & 0.00369 \\
\hline
\end{tabular}

While the density $\gamma\left[\mathrm{kg} / \mathrm{m}^{3}\right]$ and the thermal conductivity $\chi[\mathrm{Watt} / \mathrm{m} \mathrm{K}]$ of a given material are easy to obtain through simple measures, the thermal capacity $c_{p}[\mathrm{Joule} / \mathrm{kg} \mathrm{K}]$ is instead a little more complex to measure.

The theoretical/experimental procedure used there for the determination of the thermal capacity $c_{p}$ of the BBBM samples, is based on a trial and error method, and makes use of a computer program for the simulation of the thermal behaviour of a wall in non steady state by means of a finite-difference calculation.

Using this simulation program, it is possible to calculate, for each sample, the time course of the output thermal flux by using the time courses of the temperature over the faces and the time course of the input flux, all data being acquired by the computer during the test aimed to the identification of the thermal conductivity as described above.

Starting from an attempt value of $c_{p}$, comparing at any instant the time course of the output thermal flux with the experimental one using the mean square deviation as a comparison parameter, the best suited $c_{p}$ value can be obtained.

\section{Results and conclusions}

Figure 5 reports, as an example, the results obtained with the theoretical/experimental method discussed above for one specimen of BBBM material, while the table 3 resumes the mean thermo-physical characteristics of all the samples. 


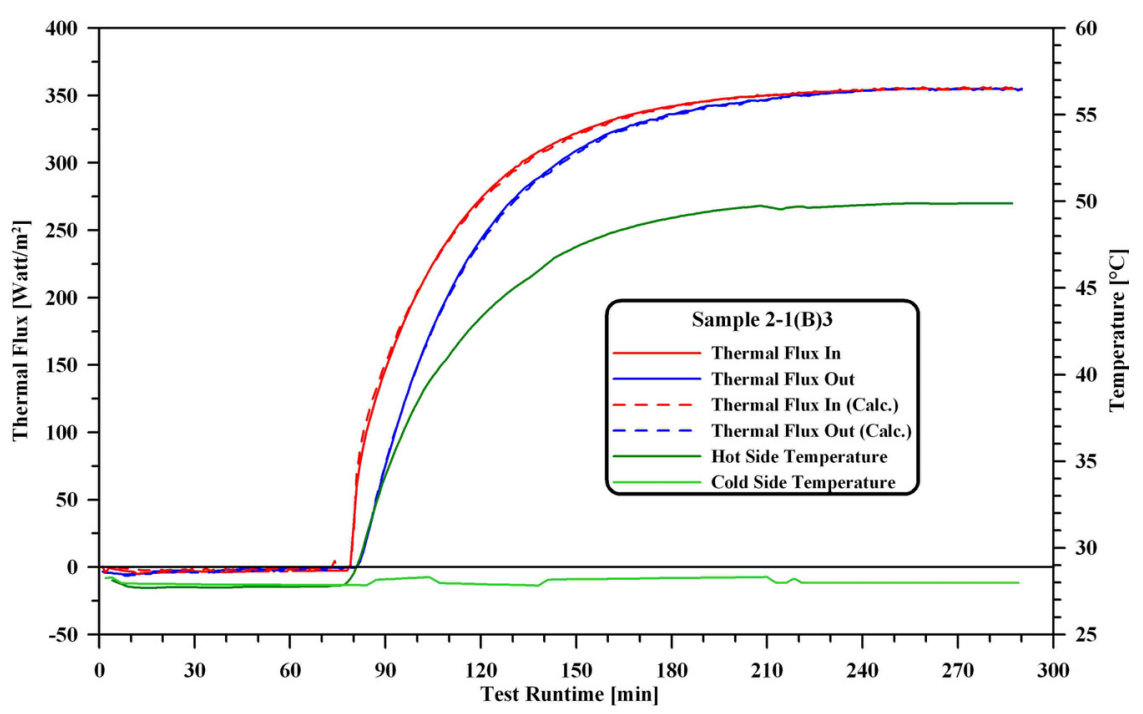

Figure 5: Test n.3 on the specimen (B) of the sample 2-1.

Table 3: Thermo-physical characteristics of the samples in dynamical state.

\begin{tabular}{|c|c|cc|c|}
\hline $\begin{array}{c}\text { Sample } \\
\text { Code }\end{array}$ & $\begin{array}{c}\% \\
\text { BBBM }\end{array}$ & $\begin{array}{c}\text { Thermal } \\
\text { conductivity } \\
{[\text { Watt/mK] }}\end{array}$ & $\begin{array}{c}\text { Thermal } \\
\text { capacity } \\
{[\mathrm{J} / \mathrm{kgK}]}\end{array}$ & $\begin{array}{c}\alpha * 10^{-7} \\
{\left[\mathrm{~m}^{2} / \mathrm{sec}\right]}\end{array}$ \\
\hline $1-3$ & 40 & 0.5384 & 1013.45 & 2.6058 \\
\hline $4-1$ & 60 & 0.2125 & 1139.71 & 1.93492 \\
\hline $11-3$ & 65 & 0.2115 & 1154.80 & 2.02048 \\
\hline $2-1$ & 70 & 0.1810 & 1178.59 & 1.85254 \\
\hline $3-2$ & 75 & 0.1725 & 1231.42 & 2.0106 \\
\hline $12-1$ & 80 & 0.1390 & 1240.69 & 1.65303 \\
\hline
\end{tabular}

The table 3 reports the thermal characteristics of all the samples (mean values over three specimens for each sample) utilized in this study and useful for the assessment of the thermal behaviour of the material in non-steady-state conditions.

Bio Based components made by using natural fibres are emerging as a realistic alternative to non bio-degradable plastic-reinforced composites, in fact they can deliver the same performances in thermal insulation and thermal capacity at lower weight and lower cost. Moreover, when used in building industry, they exhibit excellent sound absorption efficiency. 


\section{References}

[1] Chawla, K.K. \& Bastos, A.C., The mechanical properties of jute fibre and polyester/jute composites. Mechanical Behaviour of Materials, 3, pp. 191196, 1979.

[2] Bolton, A.J., Natural fibers for plastic reinforcement. Materials Technology, 9, pp. 12-20, 1994.

[3] Mohanty, A.K. \& Misra, M., Studies on Jute composites - a literature review. Polymer Plastics Technology and Engineering, 34, pp. 729-792, 1995.

[4] Lamy, B. \& Baley, C., Stiffness prediction of flax fibres/epoxy composite materials. Journal of Materials Science Letters, 19, pp. 979-980, 2000.

[5] De Albuquerque, A. C., Kuruvilla, J., de Carvalho, L.H. \& d'Almeida, J.R.M., Effect of wettability and ageing conditions on the physical and mechanical properties of uniaxially oriented jute-roving-reinforced polyester composites. Composites Science and Technology, 60(6) pp. 833844, 2000.

[6] Mohanty, A.K., Khan, Mubarak, A., Sahoo, S. \& Hinrichsen, G., Effect of chemical modification on the performance of biodegradable jute yarnBiopol composites. Journal of Materials Science, 35, pp.2589- 2595, 2000.

[7] Williams, G. I. \& Wool, R. P., Composites from natural fibres and soy oil resins. Applied Composite Materials, 7, pp. 421-432, 2000.

[8] Mohanty, A. K., Misra, M. \& Drzal, L. T., Sustainable Bio-Composites from Renewable Resources: Opportunities and Challenges in the Green Materials World. Journal of Polymers and the Environment, 10(1), pp. 1926,2002

[9] Joshi, S.V., Drzal, L.T., Mohanty, A.K. \& Arora, S., Are natural fiber composites environmentally superior to glass fiber reinforced composites?. Applied Science and Manufacturing (Incorporating Composites and Composites Manufacturing), 35(3), pp. 371-376, 2004.

[10] O'Donnell, A., Dweib, M.A. \& Wool, R.P., Natural fiber composites with plant oil-based resin. Composites Science and Technology, 64(9), pp. 1135-1145, 2004.

[11] Huda, M. S., Mohanty, A. K., Drzal, L. T., Schut, E. \& Misra, M., Green composites from recycled cellulose and poly(lactic acid): Physicomechanical and morphological properties evaluation. Journal of Materials Science, 40(16), pp. 4221-4229, 2005. 